\title{
PHYLOGENETIC ALGEBRAIC GEOMETRY
}

\author{
NICHOLAS ERIKSSON, KRISTIAN RANESTAD, BERND STURMFELS, AND SETH SULLIVANT
}

\begin{abstract}
Phylogenetic algebraic geometry is concerned with certain complex projective algebraic varieties derived from finite trees. Real positive points on these varieties represent probabilistic models of evolution. For small trees, we recover classical geometric objects, such as toric and determinantal varieties and their secant varieties, but larger trees lead to new and largely unexplored territory. This paper gives a self-contained introduction to this subject and offers numerous open problems for algebraic geometers.
\end{abstract}

\section{INTRODUCTION}

Our title is meant as a reference to the existing branch of mathematical biology which is known as phylogenetic combinatorics. By "phylogenetic algebraic geometry" we mean the study of algebraic varieties which represent statistical models of evolution. For general background reading on phylogenetics we recommend the books by Felsenstein [11] and Semple-Steel [21]. They provide an excellent introduction to evolutionary trees, from the perspectives of biology, computer science, statistics and mathematics. They also offer numerous references to relevant papers, in addition to the more recent ones listed below.

Phylogenetic algebraic geometry furnishes a rich source of interesting varieties, including familiar ones such as toric varieties, secant varieties and determinantal varieties. But these are very special cases, and one quickly encounters a cornucopia of new varieties. The objective of this paper is to give an introduction to this subject area, aimed at students and researchers in algebraic geometry, and to suggest some concrete research problems.

The basic object in a phylogenetic model is a tree $T$ which is rooted and has $n$ labeled leaves. Each node of the tree $T$ is a random variable with $k$ possible states (usually $k$ is taken to be 2 , for the binary states $\{0,1\}$, or 4 , for the nucleotides $\{A, C, G, T\})$. At the root, the distribution of the states is given by $\pi=\left(\pi_{1}, \ldots, \pi_{k}\right)$. On each edge $e$ of the tree there is a $k \times k$ transition matrix $M_{e}$ whose entries are indeterminates representing the probabilities of transition (away from the root) between the states. The random variables at the leaves are observed. The random variables at the interior nodes are hidden. Let $N$ be the total number of entries of the matrices $M_{e}$ and the vector $\pi$. These entries are called model parameters. For instance, if $T$ is a binary tree with $n$ leaves then $T$ has $2 n-2$ edges, and hence $N=(2 n-2) k^{2}+k$. In practice, there will be many constraints on these parameters, usually expressible in terms of linear equations and inequalities, so the set of statistically meaningful parameters is a polyhedron $P$ in $\mathbb{R}^{N}$. Sometimes,

1991 Mathematics Subject Classification. Primary 14Q99; Secondary 92B10.

N. Eriksson was supported by a NDSEG fellowship, K. Ranestad was supported by the Norwegian Research Council and MSRI, B. Sturmfels and S. Sullivant were partially supported by the National Science Foundation (DMS-0200729). 
these constraints are given by non-linear polynomials, in which case $P$ would be a semialgebraic subset of $\mathbb{R}^{N}$. Specifying this subset $P$ means choosing a model of evolution. Several biologically meaningful choices of such models will be discussed in Section 3.

Fix a tree $T$ with $n$ leaves. At each leaf we can observe $k$ possible states, so there are $k^{n}$ possible joint observations we can make at the leaves. The probability $\phi_{\sigma}$ of making a particular observation $\sigma$ is a polynomial in the model parameters. Hence we get a polynomial map whose coordinates are the polynomials $\phi_{\sigma}$. This map is denoted

$$
\phi: \mathbb{R}^{N} \rightarrow \mathbb{R}^{k^{n}}
$$

The map $\phi$ depends only on the tree $T$ and the number $k$. What we are interested in is the image $\phi(P)$ of this map. In real-world applications, the coordinates $\phi_{\sigma}$ represent probabilities, so they should be non-negative and sum to 1 . In other words, the rules of probability require that $\phi(P)$ lie in the standard $\left(k^{n}-1\right)$-simplex in $\mathbb{R}^{k^{n}}$. In phylogenetic algebraic geometry we temporarily abandon this requirement. We keep things simpler and closer to the familiar setting of complex algebraic geometry, by replacing $\phi$ by its complexification $\phi: \mathbb{C}^{N} \rightarrow \mathbb{C}^{k^{n}}$, and by replacing $P$ and $\phi(P)$ by their Zariski closures in $\mathbb{C}^{N}$ and $\mathbb{C}^{k^{n}}$ respectively. As we shall see, the polynomials $\phi_{\sigma}$ are often homogeneous and $\phi(P)$ is best regarded as a subvariety of a projective space.

In Section 2 we give a basic example of an evolutionary model and put it squarely in an algebraic geometric setting. This relation is then developed further in Section 3, where we describe the main families of models and show how in special cases they lead to familiar objects like Veronese and Segre varieties and their secant varieties. Section 4 is concerned with the widely used Jukes-Cantor model, which is a toric variety in a suitable coordinate system. In the last section we formulate a number of general problems in phylogenetic algebraic geometry that we find particularly important, and a list of more specific computationally oriented problems that may shed light on the more general ones.

\section{Polynomial maps DeRIVEd from a tree}

In this section we explain the polynomial map $\phi$ associated to a tree $T$ and an integer $k \geq 1$. To make things as concrete as possible, let $k=2$ and $T$ be the tree on $n=3$ leaves pictured below.

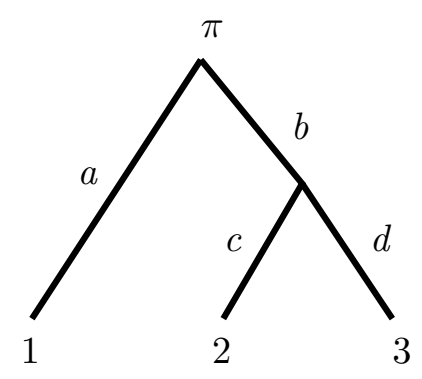

The probability distribution at the root is an unknown vector $\left(\pi_{0}, \pi_{1}\right)$. For each of the four edges of the tree, we have a $2 \times 2$-transition matrix: 


$$
\begin{array}{rlrl}
M_{a} & =\left(\begin{array}{ll}
a_{00} & a_{01} \\
a_{10} & a_{11}
\end{array}\right) & M_{b}=\left(\begin{array}{ll}
b_{00} & b_{01} \\
b_{10} & b_{11}
\end{array}\right) \\
M_{c}=\left(\begin{array}{ll}
c_{00} & c_{01} \\
c_{10} & c_{11}
\end{array}\right) & M_{d}=\left(\begin{array}{ll}
d_{00} & d_{01} \\
d_{10} & d_{11}
\end{array}\right)
\end{array}
$$

Altogether, we have introduced $N=18$ parameters, each of which represents a probability. But we regard them as unknown complex numbers. The unknown $\pi_{0}$ represents the probability of observing letter 0 at the root, and the unknown $b_{01}$ represents the probability that the letter 0 gets changed to the letter 1 along the edge $b$. All transitions are assumed to be independent events, so the monomial

$$
\pi_{u} \cdot a_{u i} \cdot b_{u v} \cdot c_{v j} \cdot d_{v k}
$$

represents the probability of observing the letter $u$ at the root, the letter $v$ at the interior node, the letter $i$ at the leaf 1 , the letter $j$ at the leaf 2 , and the letter $k$ at the leaf 3 . Now, the probabilities at the root and the interior node are hidden random variables, while the probabilities at the three leaves are observed. This leads us to consider the polynomial

$$
\phi_{i j k}=\pi_{0} a_{0 i} b_{00} c_{0 j} d_{0 k}+\pi_{0} a_{0 i} b_{01} c_{1 j} d_{1 k}+\pi_{1} a_{1 i} b_{10} c_{0 j} d_{0 k}+\pi_{1} a_{1 i} b_{11} c_{1 j} d_{1 k} .
$$

This polynomial represents the probability of observing the letter $i$ at the leaf 1 , the letter $j$ at the leaf 2 , and the letter $k$ at the leaf 3 . The eight polynomials $\phi_{i j k}$ specify our map

$$
\phi: \mathbb{C}^{18} \rightarrow \mathbb{C}^{8} .
$$

In applications, where the parameters are actual probabilities, one immediately replaces $\mathbb{C}^{18}$ by a subset $P$, for instance, the nine-dimensional cube in $\mathbb{R}^{18}$ defined by the constraints

$$
\begin{aligned}
& \pi_{0}+\pi_{1}=1, \pi_{0}, \pi_{1} \geq 0, \\
& a_{00}+a_{01}=1, a_{00}, a_{01} \geq 0, \quad a_{10}+a_{11}=1, a_{10}, a_{11} \geq 0 \\
& b_{00}+b_{01}=1, b_{00}, b_{01} \geq 0, \quad b_{10}+b_{11}=1, b_{10}, b_{11} \geq 0 \\
& c_{00}+c_{01}=1, c_{00}, c_{01} \geq 0, \quad c_{10}+c_{11}=1, c_{10}, c_{11} \geq 0 \\
& d_{00}+d_{01}=1, d_{00}, d_{01} \geq 0, \quad d_{10}+d_{11}=1, d_{10}, d_{11} \geq 0 .
\end{aligned}
$$

In phylogenetic algebraic geometry, on the other hand, we allow ourselves the luxury of ignoring inequalities and reality issues. We regard $\phi$ as a morphism of complex varieties.

The most natural thing to do, for an algebraic geometer, is to work in a projective space. The polynomials $\phi_{i j k}$ are homogeneous with respect to the different letters $a, b, c, d$ and $\pi$. We can thus change our perspective and consider our map as a projective morphism

$$
\phi: \mathbb{P}^{3} \times \mathbb{P}^{3} \times \mathbb{P}^{3} \times \mathbb{P}^{3} \times \mathbb{P}^{1} \rightarrow \mathbb{P}^{7} .
$$

This morphism is surjective, and it is an instructive undertaking to examine its fibers.

To underline the points made in the introduction, let us now cut down on the number of model parameters and replace the domain of the morphism by a natural subset $P$. For instance, let us define $P$ by requiring that the four matrices are identical

$$
M_{a}=M_{b}=M_{c}=M_{d}=\left(\begin{array}{ll}
a_{00} & a_{01} \\
a_{10} & a_{11}
\end{array}\right) .
$$


Equivalently, $P=\mathbb{P}_{\text {diag }}^{3} \times \mathbb{P}^{1}$, where $\mathbb{P}_{\text {diag }}^{3}$ is the diagonal of $\mathbb{P}^{3} \times \mathbb{P}^{3} \times \mathbb{P}^{3} \times \mathbb{P}^{3}$. The restricted morphism $\left.\phi\right|_{P}: \mathbb{P}_{\text {diag }}^{3} \times \mathbb{P}^{1} \rightarrow \mathbb{P}^{7}$ is given by the following eight polynomials:

$$
\begin{aligned}
\phi_{000} & =\pi_{0} a_{00}^{4}+\pi_{0} a_{00} a_{01} a_{10}^{2}+\pi_{1} a_{10}^{2} a_{00}^{2}+\pi_{1} a_{10}^{3} a_{11} \\
\phi_{001} & =\pi_{0} a_{00}^{3} a_{01}+\pi_{0} a_{00} a_{01} a_{10} a_{11}+\pi_{1} a_{10}^{2} a_{00} a_{01}+\pi_{1} a_{10}^{2} a_{11}^{2} \\
\phi_{010} & =\pi_{0} a_{00}^{3} a_{01}+\pi_{0} a_{00} a_{01} a_{10} a_{11}+\pi_{1} a_{10}^{2} a_{00} a_{01}+\pi_{1} a_{10}^{2} a_{11}^{2} \\
\phi_{011} & =\pi_{0} a_{00}^{2} a_{01}^{2}+\pi_{0} a_{00} a_{01} a_{11}^{2}+\pi_{1} a_{10}^{2} a_{01}^{2}+\pi_{1} a_{10} a_{11}^{3} \\
\phi_{100} & =\pi_{0} a_{00}^{3} a_{01}+\pi_{0} a_{01}^{2} a_{10}^{2}+\pi_{1} a_{11} a_{10} a_{00}^{2}+\pi_{1} a_{10}^{2} a_{11}^{2} \\
\phi_{101} & =\pi_{0} a_{00}^{2} a_{01}^{2}+\pi_{0} a_{01}^{2} a_{10} a_{11}+\pi_{1} a_{11} a_{10} a_{00} a_{01}+\pi_{1} a_{10} a_{11}^{3} \\
\phi_{110} & =\pi_{0} a_{00}^{2} a_{01}^{2}+\pi_{0} a_{01}^{2} a_{10} a_{11}+\pi_{1} a_{11} a_{10} a_{00} a_{01}+\pi_{1} a_{10} a_{11}^{3} \\
\phi_{111} & =\pi_{0} a_{01}^{3} a_{00}+\pi_{0} a_{01}^{2} a_{11}^{2}+\pi_{1} a_{11} a_{10} a_{01}^{2}+\pi_{1} a_{11}^{4} .
\end{aligned}
$$

The image of $\left.\phi\right|_{P}$ lies in the 5-dimensional projective subspace of $\mathbb{P}^{7}$ defined by $\phi_{001}=$ $\phi_{010}$ and $\phi_{101}=\phi_{110}$. It is a hypersurface of degree eight in this $\mathbb{P}^{5}$. The defining polynomial of this hypersurface has 70 terms. Studying the geometry of this fourfold is a typical problem of phylogenetic algebraic geometry. For instance, what is its singular locus?

The definition of the map $\phi$ for an arbitrary tree $T$ with $n$ leaves and an arbitrary number $k$ of states is a straightforward generalization of the $n=3$ example given above. It is simply the calculation of the probabilities of independent events along the tree. In general, each coordinate of the map $\phi$ is given by a homogeneous polynomial of degree equal to the number of edges of $T$ plus one. If the root distribution is not a parameter, the degree of these polynomials is precisely the number of edges.

One staple among the computational techniques for dealing with tree based probabilistic models is the sum-product algorithm. The sum-product algorithm is a clever application of the distributive law that allows for the fast calculation of the polynomials $\phi_{\sigma}$. The basic idea is to factor the polynomials that represent $\phi_{\sigma}$ recursively along the tree. For instance, in our example above with homogeneous rate matrix:

$$
\phi_{000}=\pi_{0} a_{00}\left(a_{00}\left(a_{00}^{2}\right)+a_{01}\left(a_{10}^{2}\right)\right)+\pi_{1} a_{10}\left(a_{10}\left(a_{00}^{2}\right)+a_{11}\left(a_{10}^{2}\right)\right) .
$$

This expression can be evaluated with 10 multiplications and 3 additions instead of the initial expression which required 16 multiplications and 3 additions. In Section 3, we will show how these factorizations help in identifying polynomial relations among the $\phi_{\sigma}$, i.e., polynomials vanishing on the image of the morphism $\phi$.

\section{Some Models and Some Familiar Varieties}

Most evolutionary models discussed in the literature have either two or four states for their random variables. The number $n$ of leaves (or taxa) can be arbitrary. Computer scientists will often concentrate on asymptotic complexity questions for $n \rightarrow \infty$, while for our purposes it would be quite reasonable to assume that $n$ is at most ten. There are no general restrictions on the underlying tree $T$, but experience has shown that trivalent trees and trees in which every leaf is at the same distance from the root are often simpler. 
Suppose now that the number $k$ of states, the number $n$ of taxa and the tree $T$ are fixed. The choice of a model is then specified by fixing a subset $P \subseteq \mathbb{C}^{N}$. The set $P$ comprises the allowed model parameters. Here is a list of commonly studied models:

General Markov: This is the model $P=\mathbb{C}^{N}$. All the transition matrices $M_{e}$ are pairwise distinct, and there are no constraints on the $k^{2}$ entries of $M_{e}$. The algebraic geometry of this model was studied by Allman and Rhodes $[1,2]$.

Group Based: The matrices $M_{e}$ are pairwise distinct, but they all have a special structure which makes them simultaneously diagonalizable by the Fourier transform of an abelian group. In particular, $P$ is a linear subspace of $\mathbb{C}^{N}$, specified by requiring that some entries of $M_{e}$ coincide with some other entries. For example, the Jukes-Cantor model for binary states $(k=2)$ stipulates that all matrices $M_{e}$ have the form $\left(\begin{array}{ll}a_{0} & a_{1} \\ a_{1} & a_{0}\end{array}\right)$. The Jukes-Cantor model for DNA $(k=4)$ is the topic of the next section. For more information on group-based models see [10, 22, 24].

Stationary Base Composition: The matrices $M_{e}$ are distinct but they all share the common left eigenvector $\pi=\left(\pi_{1}, \ldots, \pi_{k}\right)$. This hypothesis expresses the assumption that the distribution of the four nucleotides remains the same throughout some evolutionary process. An algebraic study of this model appears in [3].

Reversible: The matrices $M_{e}$ are distinct symmetric matrices with the common left eigenvector $\pi=(1,1, \ldots, 1)$. Again, as before, $P$ is a linear subspace of $\mathbb{C}^{N}$.

Commuting: The matrices $M_{e}$ are distinct but they commute pairwise. We have not yet seen this model in the biology literature, but algebraists love the commuting variety $[14,19]$. It provides a natural supermodel for the next one.

Substitution: The $M_{e}$ matrices have the form $\exp \left(t_{e} \cdot Q\right)$ where $Q$ is a fixed matrix. Equivalently, all matrices $M_{e}$ are powers of a fixed matrix $A=\exp (Q)$ with constant entries, but where the exponent $t_{e}$ is a real parameter. This is the most widely used model in biology (see [11]) but for us it has the disadvantage that it is not an algebraic variety, unless the rate matrix $Q$ has commensurate eigenvalues.

Homogeneous: The matrices $M_{e}$ are all equal, or they all belong to a small finite collection. In this model, the number of free parameters is small and independent of the tree, so the parametric inference algorithm of [18] runs in polynomial time.

No Hidden Nodes: When all nodes are observed random variables then the parameterization becomes monomial, and the model is a toric variety. For the homogeneous model, the combinatorial structure of this variety was studied in [8]

Mixture models: Suppose we are given $m$ trees $T_{1}, \ldots, T_{m}$ (not necessarily distinct) on the same set of taxa. Each tree $T_{i}$ has its own map $\phi_{i}: \mathbb{C}^{N} \rightarrow \mathbb{C}^{n^{k}}$. The mixture model is given by the sum of these maps, that is, $\phi=\phi_{1}+\cdots+\phi_{m}$. For example, the case $T_{1}=T_{2}=\cdots=T_{m}$ and $k=4$ may be used to model the fact that different regions of the genome evolve at different rates. See [12, 13].

Root distribution: For any of the above models, the root distribution $\pi$ can be taken to be uniform, $\pi=(1,1, \ldots, 1)$, or as a vector with $k$ independent entries.

Among these models are many varieties which are familiar in algebraic geometry.

Segre Varieties: These appear as a special case of the model with no hidden nodes. 
Veronese Varieties: These appear as a special case of the homogeneous model with no hidden nodes. The models in [8] are natural projections of Veronese varieties.

Toric Varieties: The previous two classes of varieties are toric. All group-based models are seen to be toric after a clever linear change of coordinates. The toric varieties of some Jukes-Cantor models will be discussed in the next section. Gröbner bases of binomials for arbitrary group-based models are given in [24].

Secant Varieties and Joins: Joins appear when taking the mixture models of a collection of models. The secant varieties of a model amounts to taking the mixture of a model with itself. A special case of the general Markov model includes the secant varieties to the Segre varieties [1]. The secant varieties to Veronese varieties [5] appear as special cases of the homogeneous models with hidden nodes.

Determinantal Varieties: Many of the evolutionary models are naturally embedded in determinantal varieties, because the tree structure imposes rank constraints on matrices derived from the probabilities observed at the leaves. Getting a better understanding of these constraints is important for both theory and practice [9].

The remainder of this section is the discussion of one example which aims to demonstrate that phylogenetic trees arise quite naturally when studying these classical objects of algebraic geometry. Consider the Segre embedding of $\mathbb{P}^{1} \times \mathbb{P}^{1} \times \mathbb{P}^{1} \times \mathbb{P}^{1}$ in $\mathbb{P}^{15}$. This four-dimensional complex manifold is given by the familiar monomial parameterization

$$
p_{i j k l}=u_{i} \cdot v_{j} \cdot w_{k} \cdot x_{l}, \quad i, j, k, l \in\{0,1\} .
$$

Its prime ideal is generated by the $2 \times 2$-minors of the following three $4 \times 4$-matrices:

$$
\left(\begin{array}{llll}
p_{0000} & p_{0001} & p_{0010} & p_{0011} \\
p_{0100} & p_{0101} & p_{0110} & p_{0111} \\
p_{1000} & p_{1001} & p_{1010} & p_{1011} \\
p_{1100} & p_{1101} & p_{1110} & p_{1111}
\end{array}\right),\left(\begin{array}{llll}
p_{0000} & p_{0001} & p_{0100} & p_{0101} \\
p_{0010} & p_{0011} & p_{0110} & p_{0111} \\
p_{1000} & p_{1001} & p_{1100} & p_{1101} \\
p_{1010} & p_{1011} & p_{1110} & p_{1111}
\end{array}\right),\left(\begin{array}{llll}
p_{0000} & p_{0010} & p_{0100} & p_{0110} \\
p_{0001} & p_{0011} & p_{0101} & p_{0111} \\
p_{1000} & p_{1010} & p_{1100} & p_{1110} \\
p_{1001} & p_{1011} & p_{1101} & p_{1111}
\end{array}\right) .
$$

These three matrices reflect the following three bracketings of the parameterization:

$$
p_{i j k l}=\left(\left(u_{i} \cdot v_{j}\right) \cdot\left(w_{k} \cdot x_{l}\right)\right)=\left(\left(u_{i} \cdot w_{k}\right) \cdot\left(v_{j} \cdot x_{l}\right)\right)=\left(\left(u_{i} \cdot x_{l}\right) \cdot\left(v_{j} \cdot w_{k}\right)\right) .
$$

And, of course, these three bracketings correspond to the three binary trees below.
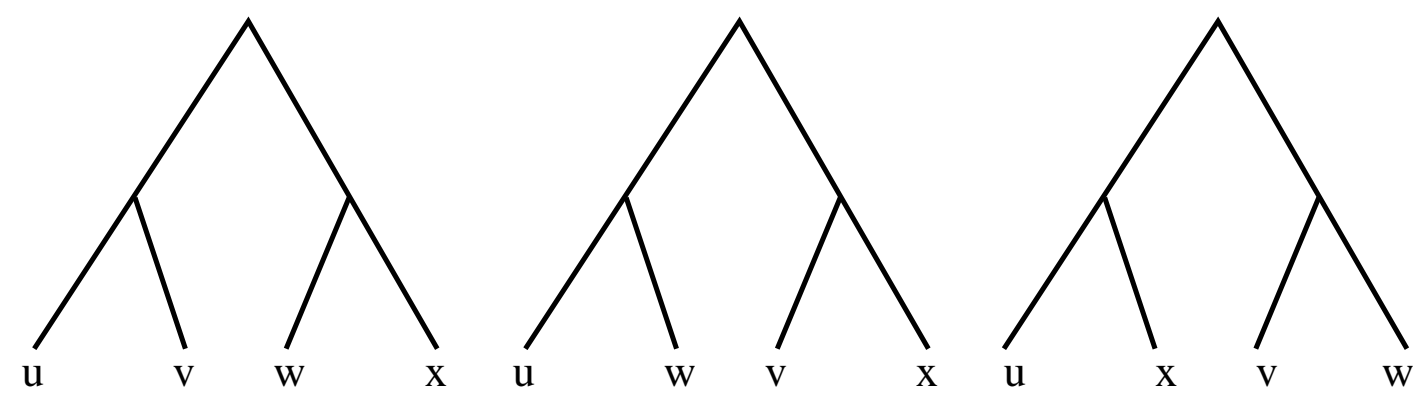

Let $X$ denote the first secant variety of the Segre variety $\mathbb{P}^{1} \times \mathbb{P}^{1} \times \mathbb{P}^{1} \times \mathbb{P}^{1}$. Thus $X$ is the nine-dimensional irreducible subvariety of $\mathbb{P}^{15}$ consisting of all $2 \times 2 \times 2 \times 2$-tensors which have tensor rank at most 2 . The secant variety $X$ has the parametric representation

$$
p_{i j k l}=\pi_{0} \cdot u_{0 i} \cdot v_{0 j} \cdot w_{0 k} \cdot x_{0 l}+\pi_{1} \cdot u_{1 i} \cdot v_{1 j} \cdot w_{1 k} \cdot x_{1 l} .
$$


This shows that the secant variety $X$ equals the general Markov model for the tree below.

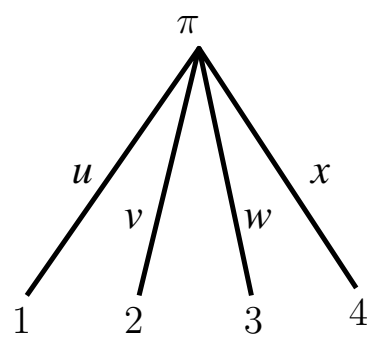

The prime ideal of $X$ is generated by all the $3 \times 3$-minors of the three matrices above. We write $X_{(12)(34)}$ for the variety defined by the $3 \times 3$-minors of the leftmost matrix, $X_{(13)(24)}$ for the variety of the $3 \times 3$-minors of the middle matrix, and $X_{(14)(23)}$ for the variety of the $3 \times 3$-minors of the rightmost matrix. Then we have, scheme-theoretically,

$$
X=X_{(12)(34)} \cap X_{(13)(24)} \cap X_{(14)(23)} .
$$

These three varieties are the general Markov models for the three binary trees depicted above. For instance, the determinantal variety $X_{(12)(34)}$ equals the general Markov model for the binary tree below.

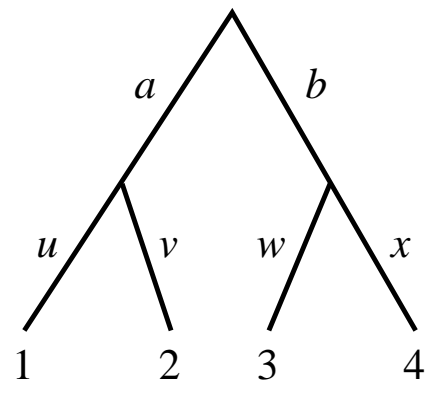

Indeed, the standard parameterization $\phi$ of this model equals

$$
\begin{aligned}
p_{i j k l}=\quad & \pi_{0} \cdot\left(a_{00} u_{0 i} v_{0 j}+a_{01} u_{1 i} v_{1 j}\right) \cdot\left(b_{00} w_{0 k} x_{0 l}+b_{01} w_{1 k} x_{1 l}\right) \\
& +\pi_{1} \cdot\left(a_{10} u_{0 i} v_{0 j}+a_{11} u_{1 i} v_{1 j}\right) \cdot\left(b_{10} w_{0 k} x_{0 l}+b_{11} w_{1 k} x_{1 l}\right) .
\end{aligned}
$$

This representation shows that the leftmost $4 \times 4$ matrix has rank at most 2 , and, conversely, every $4 \times 4$ matrix of rank $\leq 2$ can be written like this. We conclude that the general Markov model appears naturally when studying secant varieties of Segre varieties.

It is instructive to redo the above calculations under the assumption $u=v=w=x$. Then the ambient $\mathbb{P}^{15}$ gets replaced by the four-dimensional space $\mathbb{P}^{4}$ with coordinates

$$
\begin{gathered}
p_{0}=p_{0000} \\
p_{1}=p_{0001}=p_{0010}=p_{0100}=p_{1000} \\
p_{2}=p_{0011}=p_{0101}=p_{0110}=p_{1001}=p_{1010}=p_{1100} \\
p_{3}=p_{0111}=p_{1011}=p_{1101}=p_{1110} \\
p_{4}=p_{1111}
\end{gathered}
$$


Under these substitutions, all three $4 \times 4$-matrices reduce to the same $3 \times 3$-matrix

$$
\left(\begin{array}{lll}
p_{0} & p_{1} & p_{2} \\
p_{1} & p_{2} & p_{3} \\
p_{2} & p_{3} & p_{4}
\end{array}\right)
$$

The ideal of $2 \times 2$-minors now defines the rational normal curve of degree four. This special Veronese variety is the small diagonal of the Segre variety $\mathbb{P}^{1} \times \mathbb{P}^{1} \times \mathbb{P}^{1} \times \mathbb{P}^{1} \subset \mathbb{P}^{15}$. The secant variety of the rational normal curve is the cubic hypersurface in $\mathbb{P}^{4}$ defined by the determinant of the $3 \times 3$ matrix. Hence, unlike (3.1), the homogeneous model satisfies

$$
X=X_{(12)(34)}=X_{(13)(24)}=X_{(14)(23)} .
$$

Studying the stratifications of $\mathbb{P}^{n^{k}-1}$ induced by phylogenetic models, such as (3.1) and (3.2), will be one of the open problems to be presented in Section 5. First, however, let us look at some widely used models which give rise to a nice family of toric varieties.

\section{The Jukes-Cantor Model}

The Jukes-Cantor model appears frequently in the computational biology literature and represents a family of toric varieties which have the unusual property that they are not toric varieties in their natural coordinate system. Furthermore, while at first glance they sit naturally inside of $\mathbb{P}^{4^{n}-1}$, the linear span of these models involve many fewer coordinates. In this section, we will present examples of these phenomena, as well as illustrate some open problems about the underlying varieties.

Example 4.1. Let $T$ be the tree with 3 leaves below.

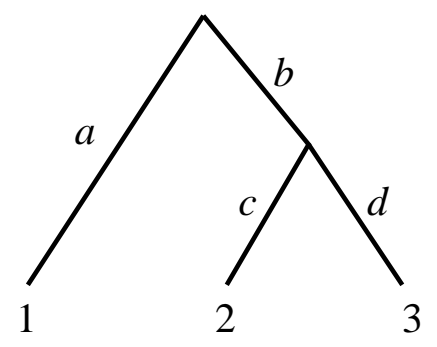

We consider the Jukes-Cantor DNA model of evolution, where each random variable has 4 states (the nucleotide bases $\mathrm{A}, \mathrm{C}, \mathrm{G}, \mathrm{T}$ ) and the root distribution is uniform, i.e., $\pi=(1 / 4,1 / 4,1 / 4,1 / 4)$. The transition matrices for the Jukes-Cantor DNA model have the form

$$
M_{a}=\left(\begin{array}{cccc}
a_{0} & a_{1} & a_{1} & a_{1} \\
a_{1} & a_{0} & a_{1} & a_{1} \\
a_{1} & a_{1} & a_{0} & a_{1} \\
a_{1} & a_{1} & a_{1} & a_{0}
\end{array}\right) .
$$

The transition matrices $M_{b}, M_{c}$, and $M_{d}$ are expressed in the same form as $M_{a}$ with " $a$ " replaced by $b, c$, and $d$ respectively. From these matrices and the rooted tree $T$, we get the map

$$
\phi: \mathbb{P}^{1} \times \mathbb{P}^{1} \times \mathbb{P}^{1} \times \mathbb{P}^{1} \rightarrow \mathbb{P}^{63}
$$


where the coordinates of $\mathbb{P}^{63}$ are the possible DNA bases at the leaves. For example,

$$
p_{A A A}=\frac{1}{4}\left(a_{0} b_{0} c_{0} d_{0}+3 a_{1} b_{1} c_{0} d_{0}+3 a_{1} b_{0} c_{1} d_{1}+3 a_{0} b_{1} c_{1} d_{1}+6 a_{1} b_{1} c_{1} d_{1}\right)
$$

That is, $p_{A A A}$ is the probability of observing the triple $A A A$ at the leaves of the tree. Since this parameterization is symmetric under renaming the bases, there are many linear relations.

$$
\begin{array}{cc}
p_{A A A}=p_{C C C}=p_{G G G}=p_{T T T} & 4 \text { terms } \\
p_{A A C}=p_{A A G}=p_{A A T}=\cdots=p_{T T G} & 12 \text { terms } \\
p_{A C A}=p_{A G A}=p_{A T A}=\cdots=p_{T G T} & 12 \text { terms } \\
p_{C A A}=p_{G A A}=p_{T A A}=\cdots=p_{G T T} & 12 \text { terms } \\
p_{A C G}=p_{A C T}=p_{A G T}=\cdots=p_{C G T} & 24 \text { terms }
\end{array}
$$

We are left with 5 distinct coordinates. From the practical standpoint, one is often interested in the accumulated coordinates, which are given parametrically as follows:

$$
\begin{gathered}
p_{123}=p_{A A A}+p_{C C C}+p_{G G G}+p_{T T T}=e_{0} c_{0} d_{0}+3 e_{1} c_{1} d_{1} \\
p_{12}=p_{A A C}+p_{A A G}+\cdots+p_{T T G}=3 e_{0} c_{0} d_{1}+3 e_{1} c_{1} d_{0}+6 e_{1} c_{1} d_{1} \\
p_{13}=p_{A C A}+p_{A G A}+\cdots+p_{T G T}=3 e_{0} c_{1} d_{0}+3 e_{1} c_{0} d_{1}+6 e_{1} c_{1} d_{1} \\
p_{23}=p_{C A A}+p_{G A A}+\cdots+p_{G T T}=3 e_{1} c_{0} d_{0}+3 e_{0} c_{1} d_{1}+6 e_{1} c_{1} d_{1} \\
p_{d i s}=p_{A C G}+p_{A C T}+\cdots+p_{C G T}=6 e_{1} c_{1} d_{0}+6 e_{1} c_{0} d_{1}+6 e_{0} c_{1} d_{1}+6 e_{1} c_{1} d_{1}
\end{gathered}
$$

where $e_{0}=a_{0} b_{0}+3 a_{1} b_{1}$ and $e_{1}=a_{0} b_{1}+a_{1} b_{0}+2 a_{1} b_{1}$. Interpreting these coordinates in terms of the probabilistic model: $p_{123}$ is the probability of seeing the same base at all three leaves, $p_{i j}$ is the probability of seeing the same base at leaves $i$ and $j$ and a different base at leaf $k$, and $p_{d i s}$ is the probability of seeing distinct bases at the three leaves.

Note that the image of $\phi$ is a three dimensional projective variety. This is a consequence of the uniform root distribution in this model. The fiber over a generic point is isomorphic to $\mathbb{P}^{1}$ and stems from the fact that it is not possible to individually determine the matrices $M_{a}$ and $M_{b}$. Only the product $M_{a} M_{b}$ can be determined. It is easily computed that the vanishing ideal of this model is generated by one cubic with 19 terms.

Remarkably, there exists a linear change of coordinates so that this polynomial becomes a binomial. Thus the corresponding variety is a toric variety in the new coordinates. This change of coordinates is given by the Fourier transform, see [24] for details. In these 
coordinates the parameterization factors:

$$
\begin{gathered}
q_{0000}=p_{123}+p_{12}+p_{13}+p_{23}+p_{\text {dis }}=\left(a_{0}+3 a_{1}\right)\left(b_{0}+3 b_{1}\right)\left(c_{0}+3 c_{1}\right)\left(d_{0}+3 d_{1}\right) \\
q_{0011}=p_{123}-\frac{1}{3} p_{12}-\frac{1}{3} p_{13}+p_{23}-\frac{1}{3} p_{d i s}=\left(a_{0}+3 a_{1}\right)\left(b_{0}+3 b_{1}\right)\left(c_{0}-c_{1}\right)\left(d_{0}-d_{1}\right) \\
q_{1101}=p_{123}-\frac{1}{3} p_{12}+p_{13}-\frac{1}{3} p_{23}-\frac{1}{3} p_{\text {dis }}=\left(a_{0}-a_{1}\right)\left(b_{0}-b_{1}\right)\left(c_{0}+3 c_{1}\right)\left(d_{0}-d_{1}\right) \\
q_{1110}=p_{123}+p_{12}-\frac{1}{3} p_{13}-\frac{1}{3} p_{23}-\frac{1}{3} p_{d i s}=\left(a_{0}-a_{1}\right)\left(b_{0}-b_{1}\right)\left(c_{0}-c_{1}\right)\left(d_{0}+3 d_{1}\right) \\
q_{1111}=p_{123}-\frac{1}{3} p_{12}-\frac{1}{3} p_{13}-\frac{1}{3} p_{23}+\frac{1}{3} p_{d i s}=\left(a_{0}-a_{1}\right)\left(b_{0}-b_{1}\right)\left(c_{0}-c_{1}\right)\left(d_{0}-d_{1}\right)
\end{gathered}
$$

In the Fourier coordinates, the cubic with nineteen terms becomes the binomial

$$
q_{0011} q_{1110} q_{1101}-q_{0000} q_{1111}^{2} \text {. }
$$

These Fourier coordinates are indexed by the subforests of the tree, where we define a subforest of a tree to be any subgraph of the tree (necessarily a forest), all of whose leaves are leaves of the original tree. For instance, the coordinate $q_{0000}$ corresponds to the empty subtree, the coordinate $q_{1101}$ corresponds to the tree from leaf 1 to leaf 3 and not including the edge to leaf 2 , and the coordinate $q_{1111}$ corresponds to the full tree on three leaves. In general there are $F_{2 n-1}$ Fourier coordinates for a tree with $n$ leaves, where $F_{m}$ is the $m$-th Fibonacci number.

Example 4.2. Now we consider an example of the Jukes-Cantor DNA model with uniform root distribution on the following tree $T$ with 4 leaves.

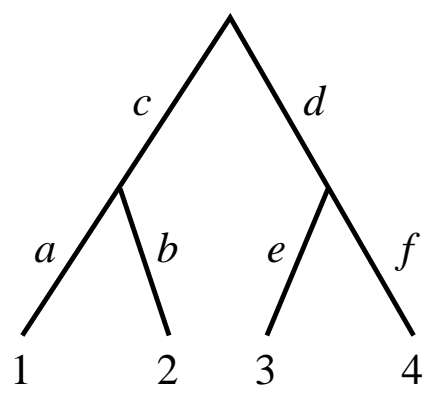

The variety of this model naturally lives in a $4^{4}-1=255$ dimensional projective space. However, after noting the symmetry of the parameterization, as in the previous example, there are only 15 coordinates in this model which are distinct. After applying the Fourier transform, the parameterization factors into a product, and hence, the variety is naturally described as a toric variety in $\mathbb{P}^{14}$. However, there are in fact 2 extra linear relations which are not simply expressed as a simple equality of probabilities so that our variety sits most naturally inside a $\mathbb{P}^{12}$. Note that $13=F_{2 \cdot 4-1}$, a Fibonacci number, as previously mentioned. We will present the parameterization in these 13 Fourier coordinates.

Associated to each of the six edges in the tree is a matrix with two parameters $\left(a_{0}\right.$ and $a_{1}, b_{0}$ and $b_{1}$, etc.) as in the previous example. The Fourier transform is a linear change 
of coordinates not only on the ambient space of the variety, but also on the parameter space. The new parametric coordinates are given by

$$
u_{0}=a_{0}+3 a_{1}, \quad u_{1}=a_{0}-a_{1}, \quad v_{0}=b_{0}+3 b_{1}, \quad v_{1}=b_{0}-b_{1}, \ldots
$$

and so on down the alphabet. To each subforest of the 4 taxa tree $T$, there is a coordinate $q_{i j k l m n}$, where the index $i j k l m n$ is the indicator vector of the edges which appear in the subforest.

The parameterization is given by the following rule

$$
q_{i j k l m n}=u_{i} \cdot v_{j} \cdot w_{k} \cdot x_{l} \cdot y_{m} \cdot z_{n} .
$$

The ideal of phylogenetic invariants in the Fourier coordinates is generated by polynomials of degrees two and three. The degree two invariants are given by the $2 \times 2$ determinants of the following matrices:

$$
\begin{gathered}
M_{0}=\left(\begin{array}{ll}
q_{000000} & q_{000011} \\
q_{110000} & q_{110011}
\end{array}\right), \\
M_{1}=\left(\begin{array}{lll}
q_{101110} & q_{101101} & q_{101111} \\
q_{011110} & q_{011101} & q_{011111} \\
q_{111110} & q_{111101} & q_{111111}
\end{array}\right) .
\end{gathered}
$$

The dimensions of these matrices are also Fibonacci numbers. The rows of these matrices are indexed by the different edge configurations to the left of the root and the columns are indexed by the edge configurations to the right of the root. There are also cubic invariants which do not have nice determinantal representations. They come in two types:

$$
q_{0000 j k} q_{1111 l m} q_{1111 n o}-q_{1100 j k} q_{1011 l m} q_{0111 n o}, \quad q_{j k 0000} q_{l m 1111} q_{n o 1111}-q_{j k 0011} q_{l m 1101} q_{n o 1110} .
$$

The only condition on $j, k, l, m, n, o$ is that each index is actually the indicator function of a subforest of the tree. The variety of the Jukes-Cantor model on a 4 taxa tree has dimension 5 , so its secant variety is a proper subvariety in $\mathbb{P}^{12}$. In applications, the secant varieties of the model are called mixture models. For this model, the secant variety has the expected dimension 11, and so is a hypersurface. Since the matrix $M_{1}$ has rank 1 on the original model, it must have rank 2 on the secant variety: thus, the desired hypersurface is the $3 \times 3$ determinant of $M_{1}$.

Example 4.3 (Determinantal closure). Now consider the Jukes-Cantor DNA model with uniform root distribution on a binary tree with 5 leaves, as pictured:

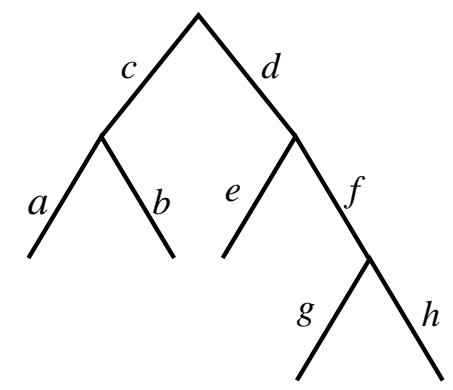


As in Example 4.1, the Fourier coordinates (modulo linear relations) are given by the subforests of $T$, of which there are 34. In the Fourier coordinates, this ideal is generated by binomials of degree 2 and 3 , of the types we have seen in the previous example. While the cubic invariants have a relatively simple description, the quadratic invariants are all represented as the $2 \times 2$ determinants of matrices naturally associated to the tree. In particular, tools from numerical linear algebra can be used to determine if these invariants are satisfied. Since the degree 2 invariants are all determinantal, it seems natural to ask what algebraic set these determinantal relations cut out: that is, what is the determinantal closure of the variety of the Jukes-Cantor DNA model on a five taxa tree? The ideal of this determinantal closure is generated by the $2 \times 2$-minors of the four following matrices:

$$
\begin{aligned}
& \left(\begin{array}{lllll}
q_{11001111} & q_{11000011} & q_{11001110} & q_{11001101} & q_{11000000} \\
q_{00001111} & q_{00000011} & q_{00001110} & q_{00001101} & q_{00000000}
\end{array}\right) \\
& \left(\begin{array}{llllllll}
q_{10111000} & q_{10110101} & q_{10110110} & q_{10111011} & q_{10110111} & q_{10111101} & q_{10111110} & q_{10111111} \\
q_{11111000} & q_{11110101} & q_{11110110} & q_{11111011} & q_{11110111} & q_{11111101} & q_{11111110} & q_{11111111} \\
q_{01111000} & q_{01110101} & q_{01110110} & q_{01111011} & q_{01110111} & q_{01111101} & q_{01111110} & q_{01111111}
\end{array}\right) \\
& \left(\begin{array}{ccccc}
q_{11111000} & q_{11000000} & q_{01111000} & q_{10011000} & q_{00000000} \\
q_{11111011} & q_{11000011} & q_{01111011} & q_{10111011} & q_{00000011}
\end{array}\right) \\
& \left(\begin{array}{lllllllll}
q_{00001101} & q_{10110101} & q_{01110101} & q_{11001101} & q_{11110101} & q_{10111101} & q_{01111101} & q_{11111101} \\
q_{00001111} & q_{10110111} & q_{01110111} & q_{11001111} & q_{11110111} & q_{10111111} & q_{01111111} & q_{11111111} \\
q_{00001110} & q_{10110110} & q_{01110110} & q_{11001110} & q_{11110110} & q_{10111110} & q_{01111110} & q_{11111110}
\end{array}\right)
\end{aligned}
$$

Surprisingly, this ideal is actually a prime ideal, and so the algebraic set is a toric variety. It has dimension 10 and degree 501, whereas this Jukes-Cantor model has only dimension 7. How does the Jukes-Cantor model sit inside its determinantal closure?

\section{Problems}

The main problem in phylogenetic algebraic geometry is to understand the complex variety, i.e., the complex Zariski closure

$$
X_{\mathbb{C}}=\overline{\phi(P)},
$$

of a phylogenetic model. This problem has many different reformulations, depending on the point of view of the person posing the problem. One problem posed by computational biologists $[6,16]$ is to determine the "phylogenetic invariants" of the model.

Problem 5.1 (Phylogenetic invariants). Find generators of the ideal defining $X_{\mathbb{C}}$.

Problem 5.2. Which equations or phylogenetic invariants are needed to distinguish between different models?

These problems are of particular interest for applications in phylogenetics, where one wishes to find which tree gives the evolutionary history of a set of taxa. Some more geometric problems are:

Problem 5.3. What are the basic geometric invariants of $\phi$ and $X_{\mathbb{C}}$ for the various models?

- What is the dimension of $X_{\mathbb{C}}$ ?

- If $\phi$ is generically finite, what is the generic degree? 
- What is the degree of $X_{\mathbb{C}}$ ?

- What is the base locus or indeterminacy locus of $\phi$ ?

- What is the singular locus of $X_{\mathbb{C}}$ ?

Problem 5.4. For a fixed type of model with $k$ states, and number $n$ of leaves (or taxa). Consider the set of rooted trees with $n$ leaves and the corresponding arrangement $\mathcal{A}$ of varieties in $\mathbb{C}^{k^{n}}$. Describe the stratification of $\mathcal{A}$, where two points in $\mathcal{A}$ are in the same strata if they are contained in the intersection of the same models. Is the stratification of $\mathcal{A}$ the same as the stratification of the space of phylogenetic trees (cf. [4])?

The tropicalization of a variety is the "logarithmic limit set" of the points on the complex variety. Tropical geometry is the geometry of the min-plus semi-ring. It was shown in [18] that the tropical geometry of statistical models plays a crucial role in parametric inference.

Problem 5.5. Determine the combinatorial structure of the tropicalizations of the various models of evolution. In particular, work out parametric inference for the substitution model.

Problem 5.6. How does the tropicalization of a mixture model relate to the tropical mixture of the tropicalization of the model: that is, compare the tropicalization of secant varieties and joins to the secant varieties and joins of tropicalizations, see [7].

In practice, it can be difficult to find a full set of generators of the ideal of $X_{\mathbb{C}}$. Therefore, we suggest certain subsets of the ideal that may be enough to distinguish between different models (as Problem 5.2 asks). We think of these subsets as types of closure operation, for example, $X_{\mathbb{C}}$ is the Zariski closure (over $\mathbb{C}$ ) of $X_{\mathbb{R}}$. We suggest the following closures as possibly easier to find and use:

Linear closure: the linear span of $X_{\mathbb{C}}$. For work on this problem, see [22].

Quadratic closure: defined by the quadratic generators of the ideal. This is closely related to the conditional independence closure from algebraic statistics, which is defined by determinantal quadratic generators, i.e., quadrics of rank 4 .

Determinantal closure: defined by the determinantal polynomials in the ideal. For example, there is a large set of determinantal relations that hold for any of the models defined above. In practice, having large sets of determinantal generators of the ideal is convenient, as determinantal conditions can be effectively evaluated using numerical linear algebra, see [9].

Local closures: defined by invariants that each depend only on subtrees of $T$. Often these give all the invariants for a model, e.g., [24].

Orbit closures: applicable if the parameter space has a dense orbit under some group and $\phi$ is equivariant. Possible related objects are quiver varieties and hyperdeterminants, see [25].

Note that part of the difficulty of studying these closure operations is coming up with a good definition for them.

Problem 5.7. Study the stratifications induced by the union of the set of "closures" of these varieties for a given model with fixed numbers of leaves (or taxa). 
¿From these rather general problems we turn to more specific, computationally-oriented problems. Many of them are special cases of the general problems above and are concrete starting points for attempting to resolve these more general problems. They also serve as an introduction to the complexity that can arise.

Problem 5.8. Consider a tree $T$ with $n$ leaves and consider the subvariety of $\left(\mathbb{C}^{2}\right)^{\otimes n}$ consisting of all $2 \times 2 \times \cdots \times 2$-tables $P$ such that all flattenings of $P$ along edges that splits $T$ have rank at most $r$. Is this variety irreducible? Do the determinants define a reduced scheme? What is the dimension of this variety?

Problem 5.9. Consider the general Markov model on a non-binary tree $T$ with 6 leaves. Is the variety $X_{\mathbb{C}}$ equal to the intersection of all models from binary trees on 6 leaves which are refinements of $T$ ? If the answer is yes, does the same statement hold schemetheoretically?

Problem 5.10. Given two trees $T$ and $T^{\prime}$ on the same number of taxa, what are the irreducible components of the intersections of their corresponding varieties?

Problem 5.11. For all trees with at most eight leaves, compute a basis for the space of linear invariants of the homogeneous Markov model, with and without hidden nodes. What about quadratic invariants?

Problem 5.12. What is the dimension of the Zariski closure of the substitution model?

Problem 5.13. Classify all phylogenetic models that are smooth.

Problem 5.14. Compute the phylogenetic complexity of the group $\mathbb{Z}_{2} \times \mathbb{Z}_{2}$ (see [24, Conjecture 28]).

Problem 5.15. Study the secant varieties of the Jukes-Cantor binary model for all trees with at most six leaves. Do any of them fail to have the expected dimension? When do determinantal conditions suffice to describe these models?

Problem 5.16. Let $T$ be the balanced binary tree on four leaves. Compute the Newton polytope (as defined in [18]) of the homogeneous model for DNA sequences.

\section{REFERENCES}

[1] E. Allman and J. Rhodes. Phylogenetic invariants for the general Markov model of sequence mutation. Mathematical Biosciences 186 (2003) 133-144.

[2] E. Allman and J. Rhodes. Quartets and parameter recovery for the general Markov model of sequence mutation, Applied Mathematics Research Express, to appear. Available at http://abacus. bates.edu/ jrhodes/

[3] E. Allman and J. Rhodes. Phylogenetic invariants for stationary base composition, Journal of Symbolic Computation, to appear. Available at http://abacus.bates.edu/ jrhodes/

[4] L. J. Billera, S. Holmes, and K. Vogtmann. Geometry of the space of phylogenetic trees. Advances in Applied Mathematics 27 (2001) 733-767.

[5] M. V. Catalisano, A. V. Geramita, and A. Gimigliano. Higher secant varieties of Segre-Veronese varieties, math. AG/0309399.

[6] J. A. Cavender and J. Felsenstein. Invariants of phylogenies: a simple case with discrete states. Journal of Classification 4 (1987) 57-71.

[7] M. Develin. Tropical secant varieties of linear spaces, 2004, math.C0/0405115, submitted to Discrete and Computational Geometry 
[8] N. Eriksson. Toric ideals of homogeneous phylogenetic models, math. C0/0401175, Proceedings of ISSAC, 149-154. ACM Press, 2004.

[9] N. Eriksson. Tree construction with singular value decomposition, in preparation, 2004. Available at http://math. berkeley.edu/ eriksson.

[10] S. Evans and T. Speed. Invariants of some probability models used in phylogenetic inference. Annals of Statistics 21 (1993) 355-377.

[11] J. Felsentein. Inferring Phylogenies. Sinauer Associates, Inc., Sunderland, 2003.

[12] V. Ferretti and D. Sankoff. Phylogenetic invariants for more general evolutionary models, Journal of Theoretical Biology 173 (1995) 147-162.

[13] V. Ferretti and D. Sankoff. A remarkable nonlinear invariant for evolution with heterogeneous rates, Mathematical Biosciences 134 (1996) 71-83.

[14] R. Guralnick, and B. Sethuraman. Commuting pairs and triples of matrices and related varieties Linear Algebra Appl. 310 (2000) 139-148.

[15] T. Hagedorn. Determining the number and structure of phylogenetic invariants, Advances in Applied Mathematics 24 (2000) 1-21.

[16] J. A. Lake. A rate-independent technique for analysis of nucleic acid sequences: evolutionary parsimony. Molecular Biology and Evolution 4 (1987) 167-191.

[17] J. M. Landsberg and L. Manivel. On the ideals of secant varieties of Segre varieties, math.AG/0311388, Foundations of Computational Mathematics, to appear.

[18] L. Pachter and B. Sturmfels. Tropical geometry of statistical models, q-bio.QM/0311009, Proceedings Natl. Acad. Sci. USA, to appear.

[19] A. Premet. Nilpotent commuting varieties of reductive Lie algebras. Invent. Math. 154 (2003) 653683

[20] D. Sankoff and M. Blanchette. Phylogenetic invariants for genome rearrangements. Journal of Computational Biology 6 (1999) 431-445.

[21] C. Semple and M. Steel. Phylogenetics. Oxford University Press, Oxford, 2003

[22] M. Steel and Y. Fu. Classifying and counting linear phylogenetic invariants for the Jukes Cantor model. Journal of Computational Biology 2 (1995) 39-47.

[23] M. Steel, L. Székely, P. Erdös, and P. Waddell. A complete family of phylogenetic invariants for any number of taxa under Kimura's 3ST model. NZ Journal of Botany 13 (1993) 289-296.

[24] B. Sturmfels and S. Sullivant. Toric ideals of phylogenetic invariants, q-bio.PE/0402015, to appear in Journal of Computational Biology.

[25] J. G. Sumner and P. D. Jarvis. Entanglement invariants and phylogenetic branching, q-bio.PE/0402007.

(N. Eriksson, S. Sullivant, and B. Sturmfels) Department of Mathematics,, University of California, Berkeley, CA 94720-3840

E-mail address, N. Eriksson: eriksson@math.berkeley.edu

E-mail address, B. Sturmfels: bernd@math.berkeley.edu

E-mail address, S. Sullivant: seths@math.berkeley.edu

(K. Ranestad) Department of Mathematics, PB 1053 Blindern, 0316 Oslo, Norway

E-mail address, K. Ranestad: ranestad@math.uio.no 